\title{
Partial discharges and bulk dielectric field enhancement
}

\author{
McAllister, lain Wilson; Johansson, Torben
}

Published in:

Annual Report Conference on Electrical Insulation and Dielectric Phenomena

Link to article, DOI:

10.1109/CEIDP.2000.883997

Publication date:

2000

Document Version

Publisher's PDF, also known as Version of record

Link back to DTU Orbit

Citation (APA):

McAllister, I. W., \& Johansson, T. (2000). Partial discharges and bulk dielectric field enhancement. In Annual Report Conference on Electrical Insulation and Dielectric Phenomena (Vol. 2, pp. 457-460).

https://doi.org/10.1109/CEIDP.2000.883997

\section{General rights}

Copyright and moral rights for the publications made accessible in the public portal are retained by the authors and/or other copyright owners and it is a condition of accessing publications that users recognise and abide by the legal requirements associated with these rights.

- Users may download and print one copy of any publication from the public portal for the purpose of private study or research.

- You may not further distribute the material or use it for any profit-making activity or commercial gain

- You may freely distribute the URL identifying the publication in the public portal

If you believe that this document breaches copyright please contact us providing details, and we will remove access to the work immediately and investigate your claim 
2000 Conference on Electrical Insulation and Dielectric Phenomena

Partial Discharges and Bulk Dielectric Field Enhancement

\author{
I. W. MCAllister and T. Johansson \\ Department of Electric Power Engineering \\ Technical University of Denmark \\ DK-2800 Lyngby \\ DENMARK
}

\begin{abstract}
A consequence of partial discharge activity within a gaseous void is the production of a field enhancement in the solid dielectric in the proximity of the void. This situation arises due to the charge created by the partial discharges accumulating at the void wall. The influence of the spatial extent of this wall charge upon the maximum field strength in the solid is examined and discussed.
\end{abstract}

\section{Introduction}

The occurrence of partial discharges in a gaseous void within a solid dielectric not only induces a charge on the detecting electrode but also leads to the accumulation of charge at the void wall. This surface charge produces an electric field which, in the solid dielectric, augments the applied field. Moreover, adjacent to the void, a significant short-range field-enhancement is manifest in the bulk medium $[1,2]$.

For spherical voids, the wall charge represents a spherical cap of charge. In the present paper, the influence of the spatial (angular) extent of the caps of charge upon the field enhancement is examined and discussed with reference to partial discharge activity.

\section{General Aspects}

We begin by considering a system of two homogeneous isotropic media $\mathbf{a}$ and $\mathbf{b}$ which possess permittivities $\varepsilon_{a}$ and $\varepsilon_{b}$ ' respectively. Medium $b$ represents a spherical inclusion, of radius $R$, embedded within medium a which occupies the remaining volume extending to infinity. Hence with respect to spherical coordinates $r, \theta, \psi$ centred on the inclusion, the interface between $\mathbf{a}$ and $\mathbf{b}$ is represented by the surface $r=R$.

At this interface we have two spherical caps of charge of opposite polarity. These are located symmetrically about a common axis, and each subtends an angle $\theta_{0}$, see Fig. 1 . The potential distributions associated with such caps of charge will now be derived.

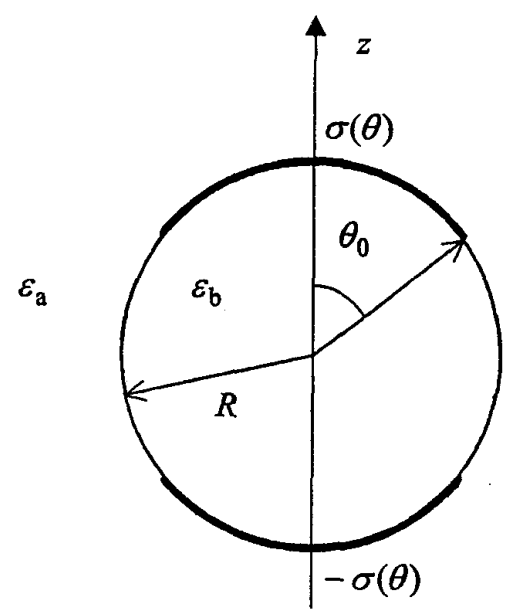

Fig.1 Geometry of spherical caps of charge.

In [3] it is shown that, for two axially symmetric elemental ring charges dq and $-\mathrm{dg}$ located at the interface between media $\mathbf{a}$ and $\mathbf{b}$ as shown in Fig.2, the associated potential functions in the 


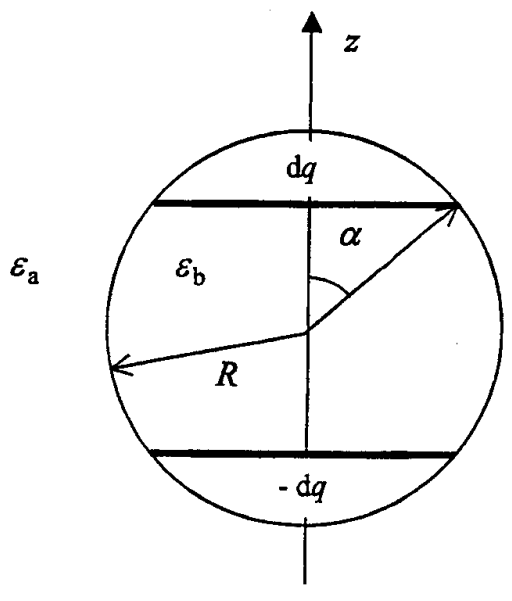

Fig.2 Location of ring charges at the interface.

two media are given by

$\mathrm{d} \phi_{\mathrm{a}}=\frac{\mathrm{dq}}{2 \pi R} \sum_{n=0}^{\infty} A_{n}\left(\frac{R}{r}\right)^{2 \mathrm{n}+2} \mathrm{P}_{2 n+1}(\cos \alpha) \times$

$\mathrm{d} \phi_{\mathrm{b}}=\frac{\mathrm{dq}}{2 \pi R} \sum_{n=0}^{\infty} A_{n}\left(\frac{r}{R}\right)^{2 n+1} \mathrm{P}_{2 n+1}(\cos \alpha) \times$

where $\alpha$ is the polar angle subtended by $d q$ and

$A_{n}=\frac{4 n+3}{(2 n+2) \varepsilon_{a}+(2 n+1) \varepsilon_{b}}$

By integrating these elemental potential functions over the spherical surface we can obtain the required solutions for $\phi_{a}$ and $\phi_{b}$.

From Fig.2, it can be readily deduced that the elemental ring charge is related to the interface charge density $\sigma(\alpha)$ by

$\mathrm{d} q=2 \pi R^{2} \sigma(\alpha) \sin \alpha \mathrm{d} \alpha$

On substitution for $d q$ into the $d \phi$ expressions, we find that the relevant integrals are expressible in the form

$I=\int_{0}^{\theta} \sigma(\alpha) \sin \alpha \mathrm{P}_{2 n+1}(\cos \alpha) \mathrm{d} \alpha$

For an initial study, we will assume that there exists a constant surface charge density, i.e. $\sigma(\alpha)=\sigma_{0}$, such that (5) reduces to

$I=\sigma_{0} \int_{0}^{\theta_{0}} \sin \alpha P_{2 n+1}(\cos \alpha) d \alpha$

Using the relationships obeyed by Legrendre polymonials, we find that

$I=\frac{\sigma_{0}}{4 n+3}\left[\mathrm{P}_{2 n}\left(\cos \theta_{0}\right)-\mathrm{P}_{2 n+2}\left(\cos \theta_{0}\right)\right]$

Consequently the associated potential distributions are

$\phi_{\mathrm{a}}=\sigma_{0} R \sum_{n=0}^{\infty} B_{n}\left(\frac{R}{r}\right)^{2 n+2} \mathrm{P}_{2 n+1}(\cos \theta) \times$
$\left.\mathrm{P}_{2 n}\left(\cos \theta_{0}\right)-\mathrm{P}_{2 n+2}\left(\cos \theta_{0}\right)\right]$
$\phi_{\mathrm{b}}=\sigma_{0} R \sum_{n=0}^{\infty} B_{n}\left(\frac{r}{R}\right)^{2 n+1} \mathrm{P}_{2 n+1}(\cos \theta) \times$
$\left[\mathrm{P}_{2 n}\left(\cos \theta_{0}\right)-\mathrm{P}_{2 n+2}\left(\cos \theta_{0}\right)\right]$

where $B_{n}$ is given by

$B_{n}=\frac{A_{n}}{4 n+3}=\frac{1}{(2 n+2) \varepsilon_{a}+(2 n+1) \varepsilon_{b}}$

Electric Field in Bulk Medium

In medium $\mathbf{a}$, the electric field along the axis of symmetry reduces simply to

$E_{a x}=-\frac{\partial \phi a}{\partial r}$

Hence upon differentiating (8) we obtain 


$$
E_{\mathrm{ax}}=\sigma_{0} \frac{\sum_{n=0}^{\infty}(2 n+2) B_{n}\left(\frac{R}{r}\right)^{2 n+3} \mathrm{P}_{2 n+1}(\cos \theta) \times}{\left[\mathrm{P}_{2 n}\left(\cos \theta_{0}\right)-\mathrm{P}_{2 n+2}\left(\cos \theta_{0}\right)\right]}
$$

For the axis of symmetry $\theta=0$ or $\theta=\pi$ and thus $P_{2 n+1}(\cos \theta)=1$ or -1 , respectively.

If we discount the non-physical singularity at the edge of the caps of charge, then the maximum field strength $E_{\text {ma }}$ in medium a will occur at the poles of the void; e.g. for $x=R$ and $\theta=0$. Hence for $\varepsilon_{b} \equiv \varepsilon_{0}$, we have

$$
\begin{aligned}
E_{\mathrm{ma}}=\frac{\sigma_{0}}{\varepsilon_{0}} \sum_{n=0}^{\infty} \frac{2 n+2}{(2 n+2) \varepsilon_{\mathrm{ra}}+2 n+1} \times \\
{\left[\mathrm{P}_{2 n}\left(\cos \theta_{0}\right)-\mathrm{P}_{2 n+2}\left(\cos \theta_{0}\right)\right] }
\end{aligned}
$$

As we are considering caps of charge with different surface areas, this implies that each cap represents a different total charge. Hence to standardize each field calculation we will employ the total charge $Q$ associated with the respective cap of charge. Total charge $Q\left(\theta_{0}\right)$ is given by

$Q=2 \pi R^{2} \int_{0}^{\theta_{0}} \sigma(\alpha) \sin \alpha \mathrm{d} \alpha$

Again for $\sigma(\alpha)=\sigma_{0}$ we have

$Q=2 \pi R^{2}\left(1-\cos \theta_{0}\right) \sigma_{0}$

Substituting for $\sigma_{0}$ enables $E_{\mathrm{ma}}$ to be expressed with respect to the associated $Q$. However to simplify further the resulting $E_{m a}$-expression, we introduce an effective field parameter $E_{Q}$ given by

$$
E_{Q}=\frac{Q}{2 \pi \varepsilon_{0} R^{2}}
$$

The variation of the dimensionless par-

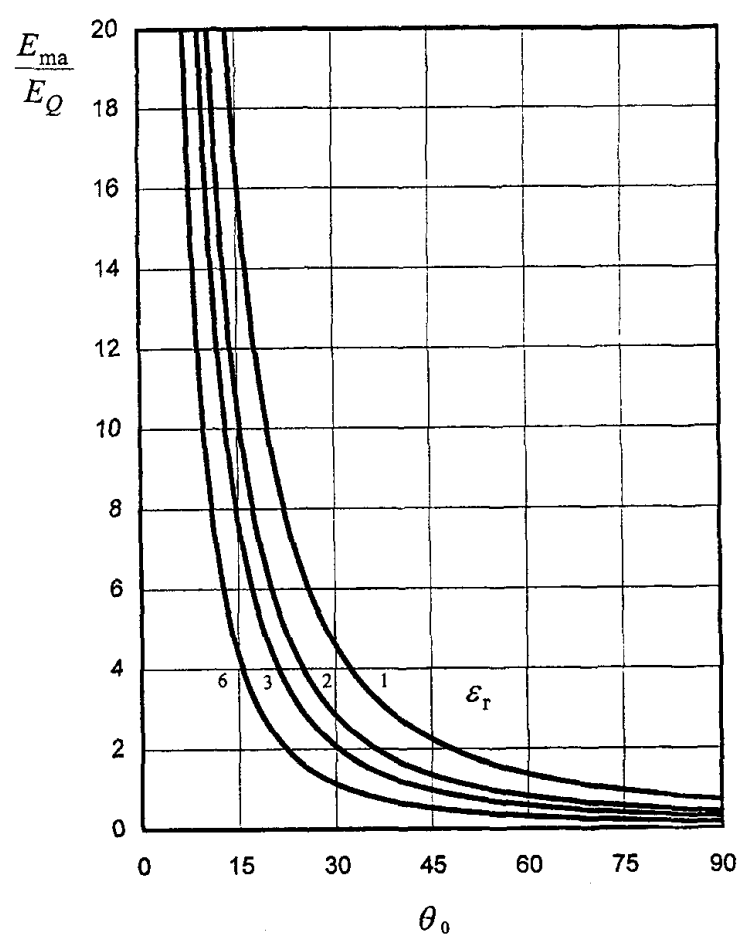

Fig.3 Variation of $E_{\mathrm{ma}} / E_{Q}$ with $\theta_{0}$.

ameter $E_{\mathrm{ma}} / E_{Q}$ is shown in Fig.3 as a function of cap angle $\theta_{0}$.

It is evident that $\theta_{0}$ has a pronounced influence upon $E_{\mathrm{ma}}$, in particular for $\theta_{0}<15^{\circ}$. In the limit, as $\theta_{0} \rightarrow 0^{\circ}$ each cap tends to a point charge of magnitude $Q$ such that $E_{\text {ma }} \rightarrow \infty$.

The influence of increasing dielectric permittivity or bulk polarisation upon reducing $E_{m a}$ is also brought into focus.

\section{Discussion and Conclusion}

The wall charge field associated with the field in the bulk dielectric augments the applied field. Thus the maximum wall charge field $E_{m a}$ reflects the level of field enhancement encountered 
in the bulk dielectric.

From the study of spherical voids, it is shown that the spatial extent of $\sigma$ has a major influence upon $E_{\text {ma }}$ for $\theta_{0}<15^{\circ}$. In contrast the influence of relative permittivity $\left(1 \leq \varepsilon_{r} \leq 6\right)$ becomes more noticeable for $\theta_{0}>15^{\circ}$.

In this initial study, the surface charge density has been taken as the independent variable. However, with reference to partial discharge activity, this is not the controlling parameter. Rather it is the internal field strength associated with the eventual quenching of the discharge development; i.e. the internal wall charge field has to accrue to such a magnitude that this process can occur.

The internal field distribution is known to be strongly influenced by the spatial extent of the wall charge, e.g. for $\theta_{0}=\pi / 2$ and $\sigma=\sigma_{0} \cos \theta$ the internal field is uniform [3]. The other limit may be taken as the field between two point charges, see [4]. With respect to the present study, the internal field is readily obtained from (9). Hence the dependence of $E_{\mathrm{ma}}$ on the spatial distribution of the wall charge for constant $Q$ must also be examined. Consequently, in future work it will be nec- essary to establish fully the relationship between the internal field and the wall charge density, prior to determining the relevant field enhancement in the bulk dielectric associated with partial discharge activity. This will obviously modify these preliminary results. However, the general validity of the conclusions reached in this study should be substantially upheld.

\section{References}

[1] A. Pedersen, G.C. Crichton \& I. W. McAllister, "PD-Related stresses in the Bulk Dielectric and Their Evaluation", 1993 Annual Report Conference on Electrical Insulation and Dielectric Phenomena, pp.474480,1993

[2] A. Pedersen, G.C. Crichton \& I. W. McAllister, "PD-Related Stresses in the Bulk Dielectric for Ellipsoidal Voids", 1994 Annual Report - Conference on Electrical Insulation and Dielectric Phenomena, pp.79-84, 1994

[3] I.W. MCAllister and G.C. Crichton, "Analysis of the Temporal Electric Fields in Lossy Dielectric Media", IEEE Trans. Elect. Insul., vol.26, pp. 513-528, 1991

[4] I.W. MCAllister, "Decay of Charge Deposited on the Wall of a Gaseous Void", IEEE Trans. Electr. Insul., vol.27, pp.1202-1207, 1992 